\title{
Entropy Solution for a Hyperbolic Equation
}

\author{
S. Bernard
}

\begin{abstract}
Nonlinear hyperbolic systems of conservation laws play a central role in Science and Engineering, and their mathematical theory as well as their numerical approximation have made recent significative progress. This paper deals with the existence and uniqueness of an entropy solution of the Cauchy problem for the quasi-linear equation $u_{t}+a(f(u))_{x}=0$ in one space dimension, where $a$ is a non-smooth coefficient.
\end{abstract}

Keywords: Conservation laws, discontinuous coefficients, product of distributions, entropy solutions

AMS subject classification: 35F25, 35L65, 35B35, 35K15

\section{Introduction}

We consider the problem

$$
\left.\begin{array}{rl}
\frac{\partial u}{\partial t}(x, t)+a(x, t) \frac{\partial}{\partial x}(f(u(x, t))) & =0 \quad(t>0) \\
u(x, 0) & =u_{0}(x)
\end{array}\right\} .
$$

This is a conservation law whose conservativity can be destroyed if $a$ is a discontinuous coefficient.

When $a$ is constant, the famous work [7] of Kruzkov leads to show the existence and uniqueness of an entropy solution, for any space dimension. This has been done in $[4,9]$, for example. Some approaches have been done in order to handle the product $a \cdot f(u)_{x}$. Namely, when $a$ satisfies a one-sided Lipschitz condition and in one space dimension, Bouchut and James gave in [3] existence, uniqueness and general stability results, for backward Lipschitz solutions and forward measure solutions, by using a duality method. Moreover, if $a$ is piecewise continuous, then they established an existence result and a precise description of the solution on the lines of discontinuity. In addition, when $a$ is bounded almost everywhere, we have built in [1] a solution of problem (1) in the space of generalized functions, where the product is well defined. But this solution is a weak one and thus is not unique. This leads us to define an entropy solution of problem (1) in order to prove then its existence and uniqueness by a "vanishing viscosity method" as in $[4,9]$.

S. Bernard: Univ. des Antilles et de la Guyane, Dép. de Math. \& Inf., Campus de Fouillole, 97159 Pointe-à-Pitre (Guadeloupe); Severine.Bernard@univ-ag.fr 
Thus, for any $\varepsilon>0$ we regularize problem (1) by Friedrichs mollification and a viscosity term which gives

$$
\left.\begin{array}{rl}
\frac{\partial u_{\varepsilon}}{\partial t}(x, t)+a_{\varepsilon}(x, t) \frac{\partial}{\partial x}\left(f_{\varepsilon}\left(u_{\varepsilon}(x, t)\right)\right)-\varepsilon \frac{\partial^{2} u_{\varepsilon}}{\partial x^{2}}(x, t) & =0 \quad(t>0) \\
u_{\varepsilon}(x, 0) & =u_{0 \varepsilon}(x)
\end{array}\right\}
$$

where $f_{\varepsilon}, a_{\varepsilon}$ and $u_{0 \varepsilon}$ are suitable regularizations of the functions $f, a$ and $u_{0}$, respectively.

We shall first study this parabolic and mollified regularization (2) and prove existence and uniqueness of a regularized solution, via standard linearized theory and a priori estimates. Thus we begin by recalling some results on function spaces and linear parabolic equations. Knowing the properties of the solution of problem (2), we will be able to pass next to the limit as $\varepsilon \rightarrow 0$. As result we will obtain a function $u$ which proves to be the entropy solution of problem (1). Uniqueness will be obtained by a slight generalization of the Kruzkov theory, that is by establishing a relation between two entropy solutions of problem (1) and their respective initial conditions.

Acknowledgement. The author thanks Professors J.-F. Colombeau and A. Méril for their advices.

\section{Function spaces and linear parabolic equations}

The study of problems in form (2) uses some results on function spaces and linear parabolic equations. In this section, we recall some of them. They can be found in [8] and we refer the reader to this book for details about their proof.

Let $T$ belong to $(0,+\infty]$ and let $X$ be a Banach space with norm $\|\cdot\|_{X}$. In the following, $\mathcal{B}(0, T, X)$ will denote the space of continuous and bounded functions from $[0, T]$ into $X$. Let us recall that for $p$ greater than 1

$$
L^{p}(0, T, X)=\left\{v:(0, T) \rightarrow X \mid\left(\int_{0}^{T}\|v(t)\|_{X} d t\right)^{\frac{1}{p}}<+\infty\right\}
$$

and

$$
L^{\infty}(0, T, X)=\left\{v:(0, T) \rightarrow X \mid \sup _{t \in(0, T)}\|v(t)\|_{X}<+\infty\right\} .
$$

Theorem 2.1 The space $W(0, T)$ defined by

$$
W(0, T)=\left\{v \in L^{2}\left(0, T, H^{1}(\mathbb{R})\right) \mid \frac{\partial v}{\partial t} \in L^{2}\left(0, T, H^{-1}(\mathbb{R})\right)\right\}
$$

satisfies the following properties:

(i) The space $\mathcal{D}\left([0, T], H^{1}(\mathbb{R})\right)$ of $\mathcal{C}^{\infty}$-functions from $[0, T]$ into $H^{1}(\mathbb{R})$ with compact support is dense in $W(0, T)$.

(ii) The inclusion $W(0, T) \subset \mathcal{B}\left(0, T, L^{2}(\mathbb{R})\right)$ holds algebraically and topologically. 
(iii) For all $u, v \in W(0, T)$ and all $t_{1}, t_{2} \in[0, T]$, the Green formula

$$
\int_{t_{1}}^{t_{2}}\left\{\left\langle\frac{\partial u}{\partial t}(\cdot, t), v(\cdot, t)\right\rangle+\left\langle u(\cdot, t), \frac{\partial v}{\partial t}(\cdot, t)\right\rangle\right\} d t=\int_{\mathbb{R}}\left\{(u v)\left(x, t_{2}\right)-(u v)\left(x, t_{1}\right)\right\} d x
$$

holds.

We end this section by reminding of classical results about linear parabolic equations of the form

$$
\left.\begin{array}{rl}
\frac{\partial u}{\partial t}-c \frac{\partial^{2} u}{\partial x^{2}}+\lambda u & =g \\
u(x, 0) & =u_{0}(x)
\end{array}\right\}
$$

where $c>0$ and $\lambda \in \mathbb{R}$ are given constants.

Theorem 2.2. Assume $u_{0} \in L^{2}(\mathbb{R})$ and $g \in L^{2}\left(0, T, H^{-1}(\mathbb{R})\right)$. Then there exists a unique solution $u \in W(0, T)$ of problem (3).

More generally, we get by induction the following regularity properties:

Theorem 2.3. Assume that, for some natural integer $m, u_{0}$ is in $H^{m}(\mathbb{R})$ and $g$ is in $L^{2}\left(0, T, H^{m-1}(\mathbb{R})\right)$. Then the solution $u$ of problem (3) satisfies
(i) $u \in L^{2}\left(0, T, H^{m+1}(\mathbb{R})\right) \cap \mathcal{B}\left(0, T, H^{m}(\mathbb{R})\right)$
(ii) $\frac{\partial u}{\partial t} \in L^{2}\left(0, T, H^{m-1}(\mathbb{R})\right)$.

\section{The viscous problem}

In connection with the regularized Cauchy problem (2) we consider the nonlinear parabolic problem

$$
\left.\begin{array}{rl}
\frac{\partial u}{\partial t}(x, t)+a(x, t) \frac{\partial}{\partial x}(f(u(x, t)))-\alpha \frac{\partial^{2} u}{\partial x^{2}}(x, t) & =0 \\
u(x, 0) & =u_{0}(x)
\end{array}\right\}
$$

where $\alpha>0$ is a constant coefficient. We first prove the existence of a solution of problem (4) by using a fixed point technique.

Lemma 3.1. Assume that $f$ is a $\mathcal{C}^{1}$-function which satisfies $M=\sup _{\xi \in \mathbb{R}}\left|f^{\prime}(\xi)\right|<$ $+\infty$ and $a \in W^{1, \infty}\left(\mathbb{R} \times \mathbb{R}_{+}\right)$. Then if $u_{0} \in L^{2}(\mathbb{R})$, problem (4) has a unique solution $u$ such that $u \in W(0, T)$ for all $T>0$.

Proof. There is no loss of generality in supposing $f(0)=0$. Now, let $\lambda>0$ be a fixed parameter. By making the change of the unknown function $v=u \exp (-\lambda t)$ we find that $u$ is a solution of problem (4) if and only if $v$ is a solution of the problem

$$
\left.\begin{array}{c}
\frac{\partial v}{\partial t}-\alpha \frac{\partial^{2} v}{\partial x^{2}}+\lambda v=-\exp (-\lambda t) \frac{\partial}{\partial x}[f(\exp (\lambda t) v)] a(x, t) \\
v(\cdot, 0)=u_{0}
\end{array}\right\} .
$$


Thus let $v \in L^{2}\left(0,+\infty, L^{2}(\mathbb{R})\right)$ be a fixed function. We consider the linear parabolic problem

$$
\left.\begin{array}{rl}
\frac{\partial w}{\partial t}-\alpha \frac{\partial^{2} w}{\partial x^{2}}+\lambda w & =-\exp (-\lambda t) \frac{\partial}{\partial x}[f(\exp (\lambda t) v)] a(x, t) \\
w(x, 0) & =u_{0}(x)
\end{array}\right\}
$$

Using the mean value theorem,

$$
\exp (-\lambda t) f(\exp (\lambda t) v) \in L^{2}\left(0,+\infty, L^{2}(\mathbb{R})\right)
$$

and therefore

$$
\exp (-\lambda t) \frac{\partial}{\partial x}[f(\exp (\lambda t) v)] \in L^{2}\left(0,+\infty, H^{-1}(\mathbb{R})\right) .
$$

But $a \in W^{1, \infty}\left(\mathbb{R} \times \mathbb{R}_{+}\right)$, so the right-hand side of (6) belongs to $L^{2}\left(0,+\infty, H^{-1}(\mathbb{R})\right)$. Hence, by applying Theorem 2.2, problem (6) has a unique solution $w \in W(0,+\infty)$.

Next denote by $F_{\lambda}$ the mapping

$$
v \in L^{2}\left(0,+\infty, L^{2}(\mathbb{R})\right) \longmapsto w=F_{\lambda}(v) \in W(0,+\infty) .
$$

We shall now prove that, for $\lambda$ large enough, $F_{\lambda}$ is a strict contraction mapping from $L^{2}\left(0,+\infty, L^{2}(\mathbb{R})\right)$ into $W(0,+\infty)$. Let $v_{i} \in L^{2}\left(0,+\infty, L^{2}(\mathbb{R})\right)$ and $w_{i}=F_{\lambda}\left(v_{i}\right) \quad(i=$ $1,2)$. Then $w=w_{1}-w_{2}$ satisfies

$$
\begin{aligned}
& \left\langle\frac{\partial w}{\partial t}(\cdot, t), z\right\rangle+\alpha \int_{\mathbb{R}} \frac{\partial w}{\partial x}(\cdot, t) \frac{\partial z}{\partial x} d x+\lambda \int_{\mathbb{R}} w(\cdot, t) z d x \\
& \quad=\exp (-\lambda t) \int_{\mathbb{R}}\left[f\left(\exp (\lambda t) v_{1}(\cdot, t)\right)-f\left(\exp (\lambda t) v_{2}(\cdot, t)\right)\right] \frac{\partial}{\partial x}[a(\cdot, t) z] d x
\end{aligned}
$$

for any $z \in H^{1}(\mathbb{R})$ and for almost any $t \in(0,+\infty)$. Let us choose $z=w(\cdot, t)$ and integrate over $(0, t)$. Since $w \in W(0,+\infty)$ and $w(0)=0$, it follows from the Green formula, the mean value theorem and the Cauchy-Schwarz inequality that

$$
\begin{gathered}
\frac{1}{2}\|w(\cdot, t)\|_{L^{2}(\mathbb{R})}^{2}+\alpha \int_{0}^{t}\left\|\frac{\partial w}{\partial x}(\cdot, s)\right\|_{L^{2}(\mathbb{R})}^{2} d s+\lambda \int_{0}^{t}\|w(\cdot, s)\|_{L^{2}(\mathbb{R})}^{2} d s \\
\leq M \int_{0}^{t}\left\|\left(v_{1}-v_{2}\right)(\cdot, s)\right\|_{L^{2}(\mathbb{R})}\left\|\frac{\partial}{\partial x}(w(\cdot, s) a(\cdot, s))\right\|_{L^{2}(\mathbb{R})} d s \\
\leq M C \int_{0}^{t}\left\|\left(v_{1}-v_{2}\right)(\cdot, s)\right\|_{L^{2}(\mathbb{R})}\|w(\cdot, s)\|_{L^{2}(\mathbb{R})} d s \\
+M C \int_{0}^{t}\left\|\left(v_{1}-v_{2}\right)(\cdot, s)\right\|_{L^{2}(\mathbb{R})}\left\|\frac{\partial w}{\partial x}(\cdot, s)\right\|_{L^{2}(\mathbb{R})} d s
\end{gathered}
$$

with $C=\|a\|_{W^{1, \infty}\left(\mathbb{R} \times \mathbb{R}_{+}\right)}$. By the inequality $x y \leq \frac{\alpha}{M C} x^{2}+\frac{M C}{4 \alpha} y^{2}$ for all $x, y \in \mathbb{R}$ we obtain

$$
\frac{1}{2}\|w(\cdot, t)\|_{L^{2}(\mathbb{R})}^{2}+(\lambda-\alpha) \int_{0}^{t}\|w(\cdot, s)\|_{L^{2}(\mathbb{R})}^{2} d s \leq \frac{M^{2} C^{2}}{2 \alpha} \int_{0}^{t}\left\|\left(v_{1}-v_{2}\right)(\cdot, s)\right\|_{L^{2}(\mathbb{R})}^{2} d s .
$$

Letting $t \rightarrow+\infty$,

$$
(\lambda-\alpha)\|w\|_{L^{2}\left(0,+\infty, L^{2}(\mathbb{R})\right)}^{2} \leq \frac{M^{2} C^{2}}{2 \alpha}\left\|v_{1}-v_{2}\right\|_{L^{2}\left(0,+\infty, L^{2}(\mathbb{R})\right)}^{2} .
$$

Hence, for $\lambda>\alpha+\frac{M^{2} C^{2}}{2 \alpha}, F_{\lambda}$ is a strict contraction mapping and has a unique fixed point $v \in L^{2}\left(0,+\infty, L^{2}(\mathbb{R})\right)$. Moreover, $v$ satisfies (5) and $v \in W(0,+\infty)$. Finally, $u=\exp (\lambda t) v \in W(0, T)$ for any $T>0$ and $u$ is the unique solution of problem (4) 
We can now prove a first theorem.

Theorem 3.1. Assume $f$ is a $\mathcal{C}^{1}$-function and $a \in W^{1, \infty}\left(\mathbb{R} \times \mathbb{R}_{+}\right)$. If $u_{0} \in L^{2}(\mathbb{R}) \cap$ $L^{\infty}(\mathbb{R})$, problem $(4)$ has a unique solution $u, u \in W(0, T) \cap L^{\infty}\left(0, T, L^{\infty}(\mathbb{R})\right)$ for all $T>0$ and

$$
\|u(\cdot, t)\|_{L^{\infty}(\mathbb{R})} \leq\left\|u_{0}\right\|_{L^{\infty}(\mathbb{R})}
$$

a.e. in $(0, T)$.

Proof. Let $\psi \in \mathcal{C}_{0}^{\infty}(\mathbb{R})$ satisfying

$$
\psi(r)= \begin{cases}1 & \text { if } r \leq\left\|u_{0}\right\|_{L^{\infty}(\mathbb{R})} \\ 0 & \text { if } r \geq\left\|u_{0}\right\|_{L^{\infty}(\mathbb{R})}+1\end{cases}
$$

We set $g=\psi f$. Now, we use Lemma 3.1 with $g$ instead of $f$. So there exists a unique function $u, u \in W(0, T)$ for any $T>0$, satisfying

$$
\left.\begin{array}{rl}
\frac{\partial u}{\partial t}+a \frac{\partial}{\partial x}[g(u)]-\alpha \frac{\partial^{2} u}{\partial x^{2}} & =0 \\
u(x, 0) & =u_{0}(x)
\end{array}\right\}
$$

Let us prove that $u$ is also a solution of problem (4). To do so, we need to check that $u$ satisfies an inequality of form (7). Let us set $v=u-\left\|u_{0}\right\|_{L^{\infty}(\mathbb{R})}$. Since

$$
\frac{\partial v}{\partial t}-\alpha \frac{\partial^{2} v}{\partial x^{2}}=\frac{\partial u}{\partial t}-\alpha \frac{\partial^{2} u}{\partial x^{2}}=-a g^{\prime}(u) \frac{\partial u}{\partial x}
$$

we have

$$
\left\langle\frac{\partial v}{\partial t}(\cdot, t), z\right\rangle+\alpha \int_{\mathbb{R}} \frac{\partial v}{\partial x}(\cdot, t) \frac{\partial z}{\partial x} d x=-\int_{\mathbb{R}} a(\cdot, t) g^{\prime}(u(\cdot, t)) \frac{\partial u}{\partial x}(\cdot, t) z d x
$$

for all $z \in H^{1}(\mathbb{R})$. In particular, for $z=v_{+}(\cdot, t)$ belonging to $H^{1}(\mathbb{R})$, since

$$
\left|v_{+}(x, t)\right|=\left|\left(u(x, t)-\left\|u_{0}\right\|_{L^{\infty}(\mathbb{R})}\right)_{+}\right| \leq|u(x, t)|
$$

for all $(x, t) \in \mathbb{R} \times \mathbb{R}_{+}$and

$$
\int_{\mathbb{R}}\left|\frac{\partial v_{+}}{\partial x}(\cdot, t)\right|^{2} d x \leq \int_{\mathbb{R}}\left|\frac{\partial u}{\partial x}(\cdot, t)\right|^{2} d x
$$

for all $t>0$, we get

$$
\begin{aligned}
\left\langle\frac{\partial v}{\partial t}(\cdot, t), v_{+}(\cdot, t)\right\rangle+\alpha \int_{\mathbb{R}} \frac{\partial v}{\partial x}(\cdot, t) \frac{\partial v_{+}}{\partial x}(\cdot, t) d x \\
=-\int_{\mathbb{R}} a(\cdot, t) g^{\prime}(u(\cdot, t)) \frac{\partial v}{\partial x}(\cdot, t) v_{+}(\cdot, t) d x
\end{aligned}
$$


Then, by integrating over $(0, T)$ and using the Green formula and the Cauchy-Schwarz inequality,

$$
\begin{aligned}
& \frac{1}{2}\left\|v_{+}(\cdot, t)\right\|_{L^{2}(\mathbb{R})}^{2} \\
& \quad \leq-\alpha \int_{0}^{t}\left\|\frac{\partial v_{+}}{\partial x}(\cdot, s)\right\|_{L^{2}(\mathbb{R})}^{2} d s+C \int_{0}^{t}\left\|v_{+}(\cdot, s)\right\|_{L^{2}(\mathbb{R})}\left\|\frac{\partial v_{+}}{\partial x}(\cdot, s)\right\|_{L^{2}(\mathbb{R})} d s
\end{aligned}
$$

where $C$ is a constant. But $-\alpha x^{2}+C x y \leq \frac{C^{2}}{4 \alpha} y^{2}$ for all $x, y \in \mathbb{R}_{+}$, so

$$
\left\|v_{+}(\cdot, t)\right\|_{L^{2}(\mathbb{R})}^{2} \leq \frac{C^{2}}{2 \alpha} \int_{0}^{t}\left\|v_{+}(\cdot, s)\right\|_{L^{2}(\mathbb{R})}^{2} d s
$$

Finally, by applying a Gronwall lemma, $\left\|v_{+}(\cdot, t)\right\|_{L^{2}(\mathbb{R})}^{2} \leq 0$, that is $v_{+}=0$. Similary, by setting $w=-u-\left\|u_{0}\right\|_{L^{\infty}(\mathbb{R})}$, we prove that $w_{+}=0$. Then $\|u(\cdot, t)\|_{L^{\infty}(\mathbb{R})} \leq\left\|u_{0}\right\|_{L^{\infty}(\mathbb{R})}$ a.e. in $(0, T)$. Therefore $u$ is a solution of problem (4) which satisfies bound (7). In order to prove uniqueness, we consider two solutions $u_{1}, u_{2} \in W(0, T) \cap L^{\infty}\left(0, T, L^{\infty}(\mathbb{R})\right)$ of problem (4). We truncate $f$ on the interval

$$
I=\left\{u \in \mathbb{R}|| u \mid \leq \max \left(\left\|u_{1}\right\|_{L^{\infty}\left(0, T, L^{\infty}(\mathbb{R})\right)},\left\|u_{2}\right\|_{L^{\infty}\left(0, T, L^{\infty}(\mathbb{R})\right)}\right)\right\}
$$

and obtain a function $g$ which derivative is bounded. But $u_{1}$ and $u_{2}$ satisfy also

$$
\left.\begin{array}{rl}
\frac{\partial u}{\partial t}+a \frac{\partial}{\partial x}[g(u)]-\alpha \frac{\partial^{2} u}{\partial x^{2}} & =0 \\
u(\cdot, 0) & =u_{0}
\end{array}\right\}
$$

By Lemma 3.1 the solution of this problem is unique, so $u_{1}=u_{2}$

Theorem 3.2. Assume that, for some integer $m \geq 0, f$ is a $\mathcal{C}^{m}$-function, $u_{0} \in$ $H^{m}(\mathbb{R}) \cap L^{\infty}(\mathbb{R})$ and $a \in W^{m, \infty}\left(\mathbb{R} \times \mathbb{R}_{+}\right)$. Then the solution u of problem (4) satisfies

$$
u \in L^{2}\left(0, T, H^{m+1}(\mathbb{R})\right) \cap \mathcal{B}\left(0, T, H^{m}(\mathbb{R})\right)
$$

for all $T>0$ and

$$
\frac{\partial^{k} u}{\partial t^{k}} \in \begin{cases}L^{2}\left(0, T, H^{m+1-2 k}(\mathbb{R})\right) \cap \mathcal{B}\left(0, T, H^{m-2 k}(\mathbb{R})\right) & \text { for } m \geq 2 k \\ L^{2}\left(0, T, L^{2}(\mathbb{R})\right) & \text { for } m=2 k-1 .\end{cases}
$$

Proof. It will be divided into several steps.

1. The case $m=1$ : We can write $\frac{\partial u}{\partial t}-\alpha \frac{\partial^{2} u}{\partial x^{2}}=-a f^{\prime}(u) \frac{\partial u}{\partial t}$. Since

$$
u \in L^{2}\left(0, T, H^{1}(\mathbb{R})\right) \cap L^{\infty}\left(0, T, L^{\infty}(\mathbb{R})\right),
$$

we have

$$
-a f^{\prime}(u) \frac{\partial u}{\partial x} \in L^{2}\left(0, T, L^{2}(\mathbb{R})\right) .
$$


Hence, applying Theorem 2.3 with $m=1, \lambda=0$ and $g=-a f^{\prime}(u) \frac{\partial u}{\partial x}$, we obtain

$$
u \in L^{2}\left(0, T, H^{2}(\mathbb{R})\right) \cap \mathcal{B}\left(0, T, H^{1}(\mathbb{R})\right) \quad \text { and } \quad \frac{\partial u}{\partial t} \in L^{2}\left(0, T, L^{2}(\mathbb{R})\right) .
$$

2. The case $m=2$ : Let us first check that

$$
v=\frac{\partial u}{\partial t} \in L^{2}\left(0, T, H^{1}(\mathbb{R})\right) \cap \mathcal{B}\left(0, T, L^{2}(\mathbb{R})\right)
$$

for all $T>0$. By differentiating (4) with respect to $t$ we obtain

$$
\frac{\partial v}{\partial t}-\alpha \frac{\partial^{2} v}{\partial x^{2}}=-a \frac{\partial}{\partial x}\left[f^{\prime}(u) v\right]+\frac{\partial a}{\partial t} \frac{\partial}{\partial x}[f(u)]
$$

Since

$$
v(\cdot, 0)=-a(\cdot, 0) f^{\prime}\left(u_{0}\right) \frac{\partial u_{0}}{\partial x}+\alpha \frac{\partial^{2} u_{0}}{\partial x^{2}} \in L^{2}(\mathbb{R})
$$

and

$$
-a \frac{\partial}{\partial x}\left[f^{\prime}(u) v\right]+\frac{\partial a}{\partial t} \frac{\partial}{\partial x}[f(u)] \in L^{2}\left(0, T, H^{-1}(\mathbb{R})\right),
$$

we can apply Theorems 2.2 and 2.1/(ii) and get

$$
\frac{\partial u}{\partial t} \in L^{2}\left(0, T, H^{1}(\mathbb{R})\right) \cap \mathcal{B}\left(0, T, L^{2}(\mathbb{R})\right) .
$$

Using the previous results and the Sobolev imbedding theorem, we obtain $\left(\frac{\partial u}{\partial x}\right)^{2} \in$ $L^{2}\left(0, T, L^{2}(\mathbb{R})\right)$. Then, by differentiating (4) with respect to $x$,

$$
\left.\begin{array}{rl}
\frac{\partial}{\partial t}\left(\frac{\partial u}{\partial x}\right)-\alpha \frac{\partial^{2}}{\partial x^{2}}\left(\frac{\partial u}{\partial x}\right) & =-\frac{\partial}{\partial x}\left[a f^{\prime}(u) \frac{\partial u}{\partial x}\right] \\
\frac{\partial u}{\partial x}(\cdot, 0) & =\frac{\partial u_{0}}{\partial x}
\end{array}\right\} .
$$

But

$$
-\frac{\partial}{\partial x}\left[a f^{\prime}(u) \frac{\partial u}{\partial x}\right] \in L^{2}\left(0, T, L^{2}(\mathbb{R})\right) \quad \text { and } \quad \frac{\partial u_{0}}{\partial x} \in H^{1}(\mathbb{R}) .
$$

So we may apply Theorem 2.3 with $m=1, \lambda=0$ and $g=-\frac{\partial}{\partial x}\left[a f^{\prime}(u) \frac{\partial u}{\partial x}\right]$, which gives

$$
\frac{\partial u}{\partial x} \in L^{2}\left(0, T, H^{2}(\mathbb{R})\right) \cap \mathcal{B}\left(0, T, H^{1}(\mathbb{R})\right) .
$$

Therefore

$$
\left.\begin{array}{rl}
u & \in L^{2}\left(0, T, H^{3}(\mathbb{R})\right) \cap \mathcal{B}\left(0, T, H^{2}(\mathbb{R})\right) \\
\frac{\partial u}{\partial t} & \in L^{2}\left(0, T, H^{1}(\mathbb{R})\right) \cap \mathcal{B}\left(0, T, L^{2}(\mathbb{R})\right)
\end{array}\right\} .
$$

3. The general case $m>2$ : By a straight forward calculation, the general case $m>2$ can be proved by induction 
We shall also require some further properties of the solution of problem (4).

Theorem 3.3. Assume that, for some integer $m \geq 3$, $f$ is a $\mathcal{C}^{m}$-function, $u_{0} \in$ $H^{m}(\mathbb{R}) \cap W^{2,1}(\mathbb{R})$ and $a \in W^{m, \infty}\left(\mathbb{R} \times \mathbb{R}_{+}\right)$. Define

$$
\begin{aligned}
A_{0} & =\|a(\cdot, 0)\|_{L^{\infty}(\mathbb{R})} \\
A & =\|a\|_{L^{\infty}\left(\mathbb{R} \times \mathbb{R}_{+}\right)} \\
M & =\sup \left\{\left|f^{\prime}(\xi)\right|: \xi \in \mathbb{R} \text { with }|\xi| \leq\left\|u_{0}\right\|_{L^{\infty}(\mathbb{R})}\right\} .
\end{aligned}
$$

Then the solution $u$ of problem (4) satisfies

$$
\begin{aligned}
\|u(\cdot, t)\|_{L^{\infty}(\mathbb{R})} & \leq\left\|u_{0}\right\|_{L^{\infty}(\mathbb{R})} \\
\left\|\frac{\partial u}{\partial x}(\cdot, t)\right\|_{L^{1}(\mathbb{R})} & \leq\left\|\frac{\partial u_{0}}{\partial x}\right\|_{L^{1}(\mathbb{R})} \\
\left\|\frac{\partial u}{\partial t}(\cdot, t)\right\|_{L^{1}(\mathbb{R})} & \leq \alpha\left\|\frac{\partial^{2} u_{0}}{\partial x^{2}}\right\|_{L^{1}(\mathbb{R})}+M\left(A+2 A_{0}\right)\left\|\frac{\partial u_{0}}{\partial x}\right\|_{L^{1}(\mathbb{R})}
\end{aligned}
$$

for all $t>0$ and

$$
\|u(\cdot, t)\|_{L^{1}(\mathbb{R})} \leq\left\|u_{0}\right\|_{L^{1}(\mathbb{R})}+M A T\left\|\frac{\partial u_{0}}{\partial x}\right\|_{L^{1}(\mathbb{R})}
$$

for all $t \in(0, T)$.

Proof. It is rather long and similar to that of [4: Theorem 2.3]. We can apply the same arguments and the coefficient function $a$ does not disturb since it belongs to $W^{m, \infty}\left(\mathbb{R} \times \mathbb{R}_{+}\right)$

\section{Existence of an entropy solution}

Let us go back to the initial problem (1). We want to use the vanishing viscosity method in order to prove the existence of an entropy solution $u$.

Let $\tau \in \mathcal{C}_{0}^{\infty}(\mathbb{R})$ be an even function, non-negative, with support contained in $[-1,1]$ and such that $\int_{\mathbb{R}} \tau(x) d x=1$. We set

$$
\begin{aligned}
f_{\varepsilon} & =f * \tau_{\varepsilon} \\
u_{0 \varepsilon} & =u_{0} * \tau_{\varepsilon} \text { with } \tau_{\varepsilon}(x)=\frac{1}{\varepsilon} \tau\left(\frac{x}{\varepsilon}\right) \quad(x \in \mathbb{R}) \\
a_{\varepsilon} & =a * \Gamma_{\varepsilon} \text { with } \Gamma_{\varepsilon}(x, y)=\tau_{\varepsilon}(x) \tau_{\varepsilon}(y) \quad\left((x, y) \in \mathbb{R} \times \mathbb{R}_{+}\right)
\end{aligned}
$$

for all $\varepsilon>0$.

Lemma 4.1. Assume $u_{0} \in L^{1}(\mathbb{R}) \cap L^{\infty}(\mathbb{R}) \cap B V(\mathbb{R})$ and $a \in W^{1, \infty}\left(\mathbb{R} \times \mathbb{R}_{+}\right)$. Then the regularized problem (2) has a unique solution $u_{\varepsilon}$ which is of $\mathcal{C}^{\infty}$-type and satisfies

$$
\begin{aligned}
\left\|u_{\varepsilon}(\cdot, t)\right\|_{L^{\infty}(\mathbb{R})} & \leq\left\|u_{0}\right\|_{L^{\infty}(\mathbb{R})} \\
\left\|\frac{\partial u_{\varepsilon}}{\partial x}(\cdot, t)\right\|_{L^{1}(\mathbb{R})} & \leq T V\left(u_{0}\right) \\
\left\|\frac{\partial u_{\varepsilon}}{\partial t}(\cdot, t)\right\|_{L^{1}(\mathbb{R})} & \leq C_{1} T V\left(u_{0}\right)
\end{aligned}
$$


for any $t \geq 0$ and

$$
\left\|u_{\varepsilon}(\cdot, t)\right\|_{L^{1}(\mathbb{R})} \leq\left\|u_{0}\right\|_{L^{1}(\mathbb{R})}+T C_{2} T V\left(u_{0}\right)
$$

for any $t \in[0, T]$, where $T V\left(u_{0}\right)$ is the total variation of $u_{0}$ and $C_{1}, C_{2}$ are constants independent on $\varepsilon$ but depending on $a$.

Proof. The functions $u_{0 \varepsilon}, f_{\varepsilon}$ and $a_{\varepsilon}$ satisfy the hypotheses of Theorems 3.2 and 3.3 for all $m \geq 0$. So problem (2) has a unique solution $u_{\varepsilon}$ which is of $\mathcal{C}^{\infty}$-type. Furthermore,

$$
\begin{aligned}
M_{\varepsilon} & =\sup \left\{\left|f_{\varepsilon}^{\prime}(\xi)\right|: \xi \in \mathbb{R} \text { with }|\xi| \leq\left\|u_{0 \varepsilon}\right\|_{L^{\infty}(\mathbb{R})}\right\} \\
& \leq \sup \left\{\left|f_{\varepsilon}^{\prime}(\xi)\right|: \xi \in \mathbb{R} \text { with }|\xi| \leq\left\|u_{0}\right\|_{L^{\infty}(\mathbb{R})}+1\right\} \quad(\text { for } \varepsilon<1) \\
& \leq \frac{C_{2}}{A}
\end{aligned}
$$

and

$$
\begin{aligned}
& A_{0}^{\varepsilon}=\left\|a_{\varepsilon}(\cdot, 0)\right\|_{L^{\infty}(\mathbb{R})} \leq A_{0} \\
& A_{\varepsilon}=\left\|a_{\varepsilon}\right\|_{L^{\infty}\left(\mathbb{R} \times \mathbb{R}_{+}\right)} \leq A .
\end{aligned}
$$

Thus we obtain the inequalities satisfied by $u_{\varepsilon}$ by applying the inequalities of Theorem 3.3 and the following properties of the function $u_{0 \varepsilon}$ :

$$
\left.\begin{array}{rl}
\left\|u_{0 \varepsilon}\right\|_{L^{1}(\mathbb{R})} & \leq\left\|u_{0}\right\|_{L^{1}(\mathbb{R})} \\
\left\|u_{0 \varepsilon}\right\|_{L^{\infty}(\mathbb{R})} & \leq\left\|u_{0}\right\|_{L^{\infty}(\mathbb{R})} \\
\left\|\frac{\partial u_{0 \varepsilon}}{\partial x}\right\|_{L^{1}(\mathbb{R})} & \leq V T\left(u_{0}\right) \\
\left\|\frac{\partial^{2} u_{0 \varepsilon}}{\partial x^{2}}\right\|_{L^{1}(\mathbb{R})} & \leq \frac{C}{\varepsilon} V T\left(u_{0}\right) \quad(C \text { is a constant independent of } \varepsilon)
\end{array}\right\}
$$

given with their proof in [4: Lemma 3.1]

Definition 4.1. We say that $u$ is an entropy solution for problem (1) if it satisfies

$$
\iint_{\mathbb{R} \times \mathbb{R}_{+}}\left[u \phi_{t}+f(u)(a \phi)_{x}\right] d x d t+\int_{\mathbb{R}} u_{0} \phi(\cdot, 0) d x=0
$$

for all $\phi \in \mathcal{D}\left(\mathbb{R} \times \mathbb{R}_{+}\right)$and, for any continuous convex entropy $E$ of flux $F$,

$$
\iint_{\mathbb{R} \times \mathbb{R}_{+}}\left[E(u) \phi_{t}+F(u)(a \phi)_{x}\right] d x d t+\int_{\mathbb{R}} E\left(u_{0}\right) \phi(\cdot, 0) d x \geq 0
$$

for all $\phi \in \mathcal{D}^{+}\left(\mathbb{R} \times \mathbb{R}_{+}\right)$, with $E(u)$ and $F(u)$ belonging to $L_{l o c}^{1}(\mathbb{R} \times(0,+\infty))$.

Remark 4.1. When $E$ is a $\mathcal{C}^{2}$-function, then $F$ is defined by the relation $F^{\prime}=f^{\prime} E^{\prime}$. But when $E$ is only continuous, $E$ is the limit, locally uniformly, of functions $E_{n}=E * \rho_{n}$, with $\rho_{n}(s)=n \rho(n s)$ for all $s \in \mathbb{R}$, where $0 \leq \rho \in \mathcal{D}(\mathbb{R})$. Since $E_{n}$ is of $\mathcal{C}^{\infty}$-type for all $n$, let $F_{n}$ be the associated flux. Thus the $F_{n}$ converge, locally uniformly, to a function $F$ which is called flux of $E$.

We are now able to prove the final result of this section. 
Theorem 4.1. Assume $u_{0} \in L^{1}(\mathbb{R}) \cap L^{\infty}(\mathbb{R}) \cap B V(\mathbb{R})$, $f$ is a $\mathcal{C}^{2}$-function and $a \in W^{1, \infty}\left(\mathbb{R} \times \mathbb{R}_{+}\right)$. Then problem (1) has an entropy solution $u$. Moreover,

$$
u \in L^{\infty}\left(0,+\infty, L^{\infty}(\mathbb{R})\right) \cap \mathcal{B}\left(0, T, L^{1}(\mathbb{R})\right)
$$

for any $T>0$ and

$$
u(\cdot, t) \in B V(\mathbb{R})
$$

for all $t \geq 0$, with

$$
\begin{aligned}
\|u(\cdot, t)\|_{L^{\infty}(\mathbb{R})} & \leq\left\|u_{0}\right\|_{L^{\infty}(\mathbb{R})} & & \text { a.e } \\
T V(u(\cdot, t)) & \leq T V\left(u_{0}\right) & & \text { for all } t \geq 0 \\
\int_{\mathbb{R}}\left|u\left(x, t_{2}\right)-u\left(x, t_{1}\right)\right| d x & \leq C T V\left(u_{0}\right)\left|t_{2}-t_{1}\right| & & \text { for } \text { all } t_{1}, t_{2} \geq 0
\end{aligned}
$$

where $C$ is a constant only depending on a.

Proof. Using the properties of each $u_{\varepsilon}$ solution of problem (2) given in Lemma 4.1, we can extract from the family of approximated solutions $\left(u_{\varepsilon}\right)_{\varepsilon>0}$ a subsequence, still denoted $\left(u_{\varepsilon}\right)_{\varepsilon>0}$, such that

$$
\left\|u_{\varepsilon}\right\|_{L^{\infty}\left(0,+\infty, L^{\infty}(\mathbb{R})\right)} \leq\left\|u_{0}\right\|_{L^{\infty}(\mathbb{R})}
$$

and

$$
\begin{aligned}
u_{\varepsilon} & \rightarrow u & & \text { in } L_{l o c}^{1}\left(0,+\infty, L_{l o c}^{1}(\mathbb{R})\right) \\
u_{\varepsilon} & \rightarrow u & & \text { a.e in } \mathbb{R} \times(0,+\infty) \\
u_{0 \varepsilon} & \rightarrow u_{0} & & \text { in } L^{1}(\mathbb{R}) \\
f_{\varepsilon}\left(u_{\varepsilon}\right) & \rightarrow f(u) & & \text { in } L_{l o c}^{1}\left(0,+\infty, L_{l o c}^{1}(\mathbb{R})\right)
\end{aligned}
$$

as $\varepsilon \rightarrow 0$, and which satisfies

$$
\iint_{\mathbb{R} \times \mathbb{R}_{+}}\left[u_{\varepsilon} \phi_{t}+f_{\varepsilon}\left(u_{\varepsilon}\right)\left(a_{\varepsilon} \phi\right)_{x}+\varepsilon u_{\varepsilon} \phi_{x x}\right] d x d t+\int_{\mathbb{R}} u_{0 \varepsilon} \phi(\cdot, 0) d x=0
$$

for all $\phi \in \mathcal{D}\left(\mathbb{R} \times \mathbb{R}_{+}\right)$and

$$
\iint_{\mathbb{R} \times \mathbb{R}_{+}}\left[E\left(u_{\varepsilon}\right) \phi_{t}+F_{\varepsilon}\left(u_{\varepsilon}\right)\left(a_{\varepsilon} \phi\right)_{x}+\varepsilon E\left(u_{\varepsilon}\right) \phi_{x x}\right] d x d t+\int_{\mathbb{R}} E\left(u_{0 \varepsilon}\right) \phi(\cdot, 0) d x \geq 0
$$

for all $\phi \in \mathcal{D}^{+}\left(\mathbb{R} \times \mathbb{R}_{+}\right)$, with $E$ a $\mathcal{C}^{2}$-convex function and $F_{\varepsilon}^{\prime}=E^{\prime} \cdot f_{\varepsilon}^{\prime}$. Since $\left(a_{\varepsilon} \phi\right)_{x} \rightarrow$ $(a \phi)_{x}$ in $L^{\infty}(\mathbb{R} \times(0,+\infty))$ as $\varepsilon \rightarrow 0$, by passing to the limit as $\varepsilon \rightarrow 0$ in (8) we obtain

$$
\iint_{\mathbb{R} \times \mathbb{R}_{+}}\left[u \phi_{t}+f(u)(a \phi)_{x}\right] d x d t+\int_{\mathbb{R}} u_{0} \phi(\cdot, 0) d x=0
$$

for all $\phi \in \mathcal{D}\left(\mathbb{R} \times \mathbb{R}_{+}\right)$. Furthermore,

$$
\left.\begin{array}{rl}
E\left(u_{\varepsilon}\right) & \rightarrow E(u) \\
E\left(u_{0 \varepsilon}\right) & \rightarrow E\left(u_{0}\right) \\
F_{\varepsilon}\left(u_{\varepsilon}\right) & \rightarrow F(u)
\end{array}\right\} \quad \text { in } L^{1}(\mathbb{R} \times(0,+\infty))
$$


as $\varepsilon \rightarrow 0$. Thus we can pass to the limit $\varepsilon \rightarrow 0$ in (9) and obtain

$$
\iint_{\mathbb{R} \times \mathbb{R}_{+}}\left[E(u) \phi_{t}+F(u)(a \phi)_{x}\right] d x d t+\int_{\mathbb{R}} E\left(u_{0}\right) \phi(\cdot, 0) d x \geq 0
$$

for all $\phi \in \mathcal{D}^{+}\left(\mathbb{R} \times \mathbb{R}_{+}\right)$and all $\mathcal{C}^{2}$-convex functions $E$.

Now, it suffices to pass from $\mathcal{C}^{2}$-convex entropies to continuous convex entropies, by specifying what the flux means in this context. Let $E$ be a continuous convex function. Then there exists $\rho \in \mathcal{D}(\mathbb{R}), \rho \geq 0$ such that $E_{n}=E * \rho_{n} \rightarrow E$ locally uniformly, as $n \rightarrow \infty$, with $\rho_{n}(s)=n \rho(n s)$ for all $s \in \mathbb{R}$. Let $F_{n}$,

$$
F_{n}(s)=\int_{0}^{s} f^{\prime}(y) E_{n}^{\prime}(y) d y \quad(s \in \mathbb{R}),
$$

be the associated flux, $F_{n} \rightarrow F$ locally uniformly as $n \rightarrow \infty$, with

$$
F(s)=f^{\prime}(s) E(s)-f^{\prime}(0) E(0)-\int_{0}^{s} f^{\prime \prime}(y) E(y) d y \quad(s \in \mathbb{R}) .
$$

But $E_{n}$ and $F_{n}$ are smooth functions such that $E_{n}$ is convex and $F_{n}^{\prime}=f^{\prime} E_{n}^{\prime}$. So

$$
\iint_{\mathbb{R} \times \mathbb{R}_{+}}\left[E_{n}(u) \phi_{t}+F_{n}(u)(a \phi)_{x}\right] d x d t+\int_{\mathbb{R}} E_{n}\left(u_{0}\right) \phi(\cdot, 0) d x \geq 0
$$

for all $\phi \in \mathcal{D}^{+}\left(\mathbb{R} \times \mathbb{R}_{+}\right)$. When $n \rightarrow \infty$, by the Lebesgue theorem,

$$
\iint_{\mathbb{R} \times \mathbb{R}_{+}}\left[E(u) \phi_{t}+F(u)(a \phi)_{x}\right] d x d t+\int_{\mathbb{R}} E\left(u_{0}\right) \phi(\cdot, 0) d x \geq 0
$$

for all $\phi \in \mathcal{D}^{+}\left(\mathbb{R} \times \mathbb{R}_{+}\right)$and all continuous convex function E. Thus $u$ is an entropy solution of problem (1) which satisfies $\|u\|_{L^{\infty}(\mathbb{R} \times(0,+\infty))} \leq\left\|u_{0}\right\|_{L^{\infty}(\mathbb{R})}$. The proof of the other inequalities is rather long and not different from the one of [4: Theorem 3.1]. The idea is to show them for $u_{\varepsilon}$ and then pass to the limit as $\varepsilon \rightarrow 0$

\section{Uniqueness of the entropy solution}

Now it remainds to prove uniqueness of this entropy solution. For this we slightly generalize the Kruzkov theory. Indeed, we give an equivalent definition of an entropy solution of problem (1) and we use this last one to get an inequality between two entropy solutions and their respective initial conditions. Let $T>0$.

Lemma 5.1. A bounded measurable function $u$ on $\mathbb{R} \times(0, T)$ is an entropy solution of problem (1) if and only if it satisfies

$$
\begin{aligned}
\int_{\mathbb{R}} \int_{0}^{T}\left[|u-k| \phi_{t}+[f(u)-f(k)]\right. & \left.\operatorname{sgn}(u-k)(a \phi)_{x}\right] d x d t \\
& +\int_{\mathbb{R}}\left|u_{0}-k\right| \phi(\cdot, 0) d x \geq 0
\end{aligned}
$$


for all $k \in \mathbb{R}$ and all $\phi \in \mathcal{D}^{+}(\mathbb{R} \times(0, T))$.

Proof. If $u$ is an entropy solution of problem (1), then

$$
\iint_{\mathbb{R} \times \mathbb{R}_{+}}\left[E(u) \phi_{t}+F(u)(a \phi)_{x}\right] d x d t+\int_{\mathbb{R}} E\left(u_{0}\right) \phi(\cdot, 0) d x \geq 0
$$

for all $\phi \in \mathcal{D}^{+}\left(\mathbb{R} \times \mathbb{R}_{+}\right)$and all continuous convex functions $E$ of flux $F$. In particular, for $E(u)=|u-k|$ we have $F(u)=[f(u)-f(k)] \operatorname{sgn}(u-k)$. So we obtain (10) for all $k \in \mathbb{R}$.

Conversely, let $u \in L^{\infty}(\mathbb{R} \times(0, T))$ verifying (10). Then there exist $p, q \in \mathbb{R}$ such that the functions $u$ and $u_{0}$ take their values in $(p, q)$. We apply (10) with $k=p$ and then with $k=q$ concluding

$$
\int_{\mathbb{R}} \int_{0}^{T}\left[u \phi_{t}+f(u)(a \phi)_{x}\right] d x d t+\int_{\mathbb{R}} u_{0} \phi(\cdot, 0) d x=0
$$

for all $\phi \in \mathcal{D}^{+}(\mathbb{R} \times(0, T))$, and this is still true without sign condition on $\phi$, and for all $T>0$. Let $E$ be a continuous convex entropy of flux $F$. There exists $\gamma>0$ and an entropy $E_{\gamma}$ of flux $F_{\gamma}$ verifying

$$
\left.\begin{array}{rlrl}
E(s) & \leq E_{\gamma}(s) \leq E(s)+\gamma & & \text { for all } s \in[p, q] \\
E_{\gamma}(s) & =b_{0}+b_{1} s+\sum_{j} a_{j}\left|s-k_{j}\right| & & \text { for all } s \in[p, q]
\end{array}\right\}
$$

with $a_{j}>0$ and $k_{j} \in \mathbb{R}$. Then

$$
F_{\gamma}(s)=b_{0} f(s)+\sum_{j} a_{j}\left[f(s)-f\left(k_{j}\right)\right] \operatorname{sgn}\left(u-k_{j}\right) .
$$

For $\phi \in \mathcal{D}^{+}(\mathbb{R} \times(0, T))$ we have

$$
\begin{aligned}
A_{\gamma}= & \int_{\mathbb{R}} \int_{0}^{T}\left[E_{\gamma}(u) \phi_{t}+F_{\gamma}(u)(a \phi)_{x}\right] d x d t \\
& +\int_{\mathbb{R}} E_{\gamma}\left(u_{0}\right) \phi(\cdot, 0) d x \\
= & \sum_{j} a_{j}\left[\int_{\mathbb{R}} \int_{0}^{T}\left[\left|u-k_{j}\right| \phi_{t}+\left[f(u)-f\left(k_{j}\right)\right] \operatorname{sgn}\left(u-k_{j}\right)(a \phi)_{x}\right] d x d t\right. \\
& \left.+\int_{\mathbb{R}}\left|u_{0}-k_{j}\right| \phi(\cdot, 0) d x\right] \\
\geq & 0 .
\end{aligned}
$$

Since $E_{\gamma} \rightarrow E$ and $F_{\gamma} \rightarrow F$ uniformly when $\gamma \rightarrow 0$ and $a \in W^{1, \infty}\left(\mathbb{R} \times \mathbb{R}_{+}\right)$,

$$
\int_{\mathbb{R}} \int_{0}^{T}\left[E(u) \phi_{t}+F(u)(a \phi)_{x}\right] d x d t+\int_{\mathbb{R}} E\left(u_{0}\right) \phi(\cdot, 0) d x \geq 0
$$

for all $\phi$ in $\mathcal{D}^{+}(\mathbb{R} \times(0, T))$ and all $T>0$ 
Next, we prove the following basic result:

Lemma 5.2. Assume $a \in W^{1, \infty}\left(\mathbb{R} \times \mathbb{R}_{+}\right)$. Let $u$ and $v$ be two entropy solutions of problem (1) with initial conditions $u_{0}$ and $v_{0}$, respectively. Then

$$
\begin{aligned}
\int_{\mathbb{R}} \int_{0}^{T}\left[|u-v| \phi_{t}+[f(u)-f(v)]\right. & \left.\operatorname{sgn}(u-v)(a \phi)_{x}\right] d x d t \\
& +\int_{\mathbb{R}}\left|u_{0}-v_{0}\right| \phi(\cdot, 0) d x \geq 0
\end{aligned}
$$

for all $\phi$ in $\mathcal{D}^{+}(\mathbb{R} \times(0, T))$.

Proof. Let $Q=\mathbb{R} \times(0, T)$ and $\varphi \in \mathcal{D}^{+}(Q \times Q)$. We apply (10) with the entropy solution $u, k=v(y, s)$ and the test function $\varphi(\cdot, \cdot, y, s)$ and integrate with respect to $(y, s)$ on $Q$. Then we do the same computation by inverting $u$ and $v$, with the test function $\varphi(x, t, \cdot, \cdot)$. By summing the two inequalities obtained, we have

$$
\begin{aligned}
0 \leq & \int_{Q} \int_{Q} \mid u(x, t)-v(y, s)\left(\varphi_{t}+\varphi_{s}\right)(x, t, y, s) d x d t d y d s \\
& +\int_{Q} \int_{Q} \operatorname{sgn}(u(x, t)-v(y, s))[f(u(x, t))-f(v(y, s))] \\
& \times\left[[a(x, t) \varphi(x, t, y, s)]_{x}+[a(y, s) \varphi(x, t, y, s)]_{y}\right] d x d t d y d s \\
& +\int_{\mathbb{R}} \int_{Q}\left|u_{0}(x)-v(y, s)\right| \varphi(x, 0, y, s) d x d y d s \\
& +\int_{\mathbb{R}} \int_{Q}\left|v_{0}(y)-u(x, t)\right| \varphi(x, t, y, 0) d x d t d y .
\end{aligned}
$$

Let $\phi \in \mathcal{D}^{+}(Q)$. We apply (12) with

$$
\varphi_{\varepsilon}(x, t, y, s)=\phi(x, t) \chi_{\varepsilon}(x-y, t-s)
$$

where $\chi_{\varepsilon}(x, t)=\frac{1}{\varepsilon^{2}} \chi\left(\frac{x}{\varepsilon}, \frac{t}{\varepsilon}\right)$ is a non-negative approximation of the Dirac delta function at the origin, $\chi \in \mathcal{D}^{+}\left(\mathbb{R}^{2}\right)$ with $\int_{\mathbb{R}^{2}} \chi(x, y) d x d y=1$. We choose $\chi(x, t)=\theta(x) \eta(t), \eta$ with support in $(-2,-1)$. Then $(12)$ becomes

$$
\begin{aligned}
0 \leq & \int_{Q} \int_{Q}|u(x, t)-v(y, s)| \phi_{t}(x, t) \chi_{\varepsilon}(x-y, t-s) d x d t d y d s \\
& +\int_{Q} \int_{Q}[f(u(x, t))-f(v(y, s))] \operatorname{sgn}(u(x, t)-v(y, s)) \\
& \times\left[a_{x}(x, t)+a_{y}(y, s)\right] \phi(x, t) \chi_{\varepsilon}(x-y, t-s) d x d t d y d s \\
& +\int_{Q} \int_{Q}[f(u(x, t))-f(v(y, s))] \operatorname{sgn}(u(x, t)-v(y, s))
\end{aligned}
$$




$$
\begin{aligned}
& \times a(x, t) \phi_{x}(x, t) \chi_{\varepsilon}(x-y, t-s) d x d t d y d s \\
& -\int_{Q} \int_{Q} \frac{\partial}{\partial x}[[f(u(x, t))-f(v(y, s))] \operatorname{sgn}(u(x, t)-v(y, s))] \\
& \times[a(x, t)-a(y, s)] \phi(x, t) \chi_{\varepsilon}(x-y, t-s) d x d t d y d s \\
& -\int_{Q} \int_{Q}[f(u(x, t))-f(v(y, s))] \operatorname{sgn}(u(x, t)-v(y, s)) \\
& \times a_{x}(x, t) \phi(x, t) \chi_{\varepsilon}(x-y, t-s) d x d t d y d s \\
& -\int_{Q} \int_{Q}[f(u(x, t))-f(v(y, s))] \operatorname{sgn}(u(x, t)-v(y, s)) \\
& \times[a(x, t)-a(y, s)] \phi_{x}(x, t) \chi_{\varepsilon}(x-y, t-s) d x d t d y d s \\
& +\int_{\mathbb{R}} \int_{Q}\left|u_{0}(x)-v(y, s)\right| \phi(x, 0) \chi_{\varepsilon}(x-y,-s) d x d y d s \\
& +\int_{\mathbb{R}} \int_{Q}\left|v_{0}(y)-u(x, t)\right| \phi(x, t) \chi_{\varepsilon}(x-y, t) d x d t d y .
\end{aligned}
$$

Pass to the limit as $\varepsilon \rightarrow 0$, we obtain (11) by using the following convergence result which proof is in [9: Lemma 2.7.2] and the fact that $\operatorname{supp} \eta \subset(-2,-1)$

Lemma 5.3. Let $F$ be a locally Lipschitz function on $\mathbb{R}^{2}$. Then, for all $\varphi_{\varepsilon}$ in form (13), as $\varepsilon \rightarrow 0$ :

(i) The integral $\int_{Q} \int_{Q} F(u(x, t), v(y, s)) \varphi_{\varepsilon}(x, t, y, s) d x d t d y d s$ tends to the integral $\int_{Q} F(u(x, t), v(x, t)) \phi(x, t) d x d t$.

(ii) The integral $\int_{\mathbb{R}} \int_{Q} F\left(u_{0}(x), v(y, s)\right) \varphi_{\varepsilon}(x, 0, y, s) d x d y d s$ tends to the integral $\int_{Q} F\left(u_{0}(x), v_{0}(x)\right) \phi(x, 0) d x$.

Proposition 5.1. Assume $a \in W^{1, \infty}\left(\mathbb{R} \times \mathbb{R}_{+}\right)$and let $u$ and $v$ be two entropy solutions of problem (1) with initial conditions $u_{0}$ and $v_{0}$, respectively. Then, for all $s \geq 0$ and all $c<b$,

$$
\int_{c}^{b}|u(x, s)-v(x, s)| d x \leq \int_{c-M A s}^{b+M A s}\left|u_{0}(x)-v_{0}(x)\right| d x
$$

where $A=\|a\|_{L^{\infty}\left(\mathbb{R} \times \mathbb{R}_{+}\right)}, M=\sup _{r \in I}\left|f^{\prime}(r)\right|$ and $I$ is the smallest interval containing the bounded values of $u$ and $v$.

Proof. Let

$$
B=\{(x, t) \in \mathbb{R} \times[0, T]: c-M A(s-t)<x<b+M A(s-t)\}
$$

and

$$
B_{t}=\{x \in \mathbb{R}:(x, t) \in B\}
$$

for all $t \in[0, T]$. By translation we can assume that $c=-b$. Let $d>b$ and let us choose $\theta \in \mathcal{D}^{+}(\mathbb{R})$ decreasing such that

$$
\theta(y)= \begin{cases}1 & \text { if }|y|<b \\ 0 & \text { if }|y|>d\end{cases}
$$


and $\chi \in \mathcal{D}^{+}(-\infty, T)$ such that $\chi(0)=1$. We apply (11) with

$$
\phi(x, t)=\chi(t) \theta(|x|+M A(t-s))
$$

which gives

$$
\begin{aligned}
0 \leq & \int_{\mathbb{R}} \int_{0}^{T} \chi^{\prime}(t) \theta(|x|+M A(t-s))|u-v| d x d t \\
& +\int_{\mathbb{R}} \int_{0}^{T}[f(u)-f(v)] \operatorname{sgn}(u-v) a_{x} \chi(t) \theta(|x|+M A(t-s)) d x d t \\
& +\int_{\mathbb{R}}\left|u_{0}-v_{0}\right| \theta(|x|-M A s) d x
\end{aligned}
$$

since $\chi$ is non-negative and $\theta^{\prime}$ is negative. Then, letting $d \rightarrow b$,

$$
0 \leq \int_{0}^{T} \chi^{\prime}(t) \int_{B_{t}}|u-v| d x d t+\int_{B_{0}}\left|u_{0}-v_{0}\right| d x+2 M A\left\|u_{0}\right\|_{L^{\infty}(\mathbb{R})} \int_{0}^{T} \chi(t)\left|B_{t}\right| d t
$$

After setting $h(t)=\int_{B_{t}}|u(x, t)-v(x, t)| d x$ for all $t \in[0, T]$, integrating by parts and changing variables we obtain

$$
\begin{aligned}
0 & \leq-\int_{0}^{T} \chi(t) h^{\prime}(t) d t-4\left\|u_{0}\right\|_{L^{\infty}(\mathbb{R})} \int_{b+M A s}^{b+M A(s-T)} \chi\left(\frac{b+M A s-y}{M A}\right) y d y \\
& \leq-\int_{0}^{T} \chi(t) h^{\prime}(t) d t
\end{aligned}
$$

since $\chi$ is a non-negative function. But $u, v \in \mathcal{B}\left(0, T, L^{1}(\mathbb{R})\right)$, so $h$ is continuous and decreasing over $[0, T]$. We obtain the requiered inequality for $c=-b$ by writting that $h(0) \leq h(s)$ for all $s \in[0, T]$

As an immediate consequence of Theorem 4.1 and Proposition 5.1, we have the following uniqueness result:

Theorem 5.1. Assume that the functions $u_{0}, f$ and a satisfy the hypotheses of Theorem 4.1. Then the entropy solution of problem (1) is unique.

Proof. Let us assume that there exists two entropy solutions $u$ and $v$ of problem (1) with the same initial condition $u_{0}$. Applying Proposition 5.1 with $b=-c=R>0$, we obtain $\int_{B(0, R)}|u(x, t)-v(x, t)| d x=0$ for all $t \geq 0$. Since $u$ and $v$ belong to $\mathcal{B}\left(0, T, L^{1}(\mathbb{R})\right)$, when $R \rightarrow \infty$ we get $\int_{\mathbb{R}}|u(x, t)-v(x, t)| d x=0$ for all $t \geq 0$. Thus $u=v$ 


\section{S. Bernard}

\section{The main existence and uniqueness result}

Let us assume that the functions $f$ and $a$ satisfy the hypotheses of Theorem 4.1. In this section we prove the existence and uniqueness of an entropy solution of problem (1) in the general case of an initial condition $u_{0} \in L^{\infty}(\mathbb{R})$.

We introduce the mapping $S: u_{0} \longmapsto u=S u_{0}$, which associates with the initial condition $u_{0} \in L^{1}(\mathbb{R}) \cap L^{\infty}(\mathbb{R}) \cap B V(\mathbb{R})$ the entropy solution $u$ of problem (1) given by Theorem 4.1. We set $u(\cdot, t)=S(t) u_{0}$.

Let us first state:

Lemma 6.1. The mapping $S$ maps the space $L^{1}(\mathbb{R}) \cap L^{\infty}(\mathbb{R}) \cap B V(\mathbb{R})$ into the space $L^{1}(\mathbb{R} \times(0,+\infty)) \cap \mathcal{B}\left(0,+\infty, L^{1}(\mathbb{R})\right)$. Moreover, if $u_{0}, v_{0} \in L^{1}(\mathbb{R}) \cap L^{\infty}(\mathbb{R}) \cap B V(\mathbb{R})$, then

$$
\left\|S(t) u_{0}-S(t) v_{0}\right\|_{L^{1}(\mathbb{R})} \leq\left\|u_{0}-v_{0}\right\|_{L^{1}(\mathbb{R})}
$$

for all $t \geq 0$.

As a consequence of this lemma, we have:

Theorem 6.1. The mapping $S$ may be extended by continuity to a uniformly continuous mapping from $L^{1}(\mathbb{R})$ into $\mathcal{B}\left(0,+\infty, L^{1}(\mathbb{R})\right)$. In addition, for all $t \geq 0$, the mapping

$$
S(t): L^{1}(\mathbb{R}) \rightarrow L^{1}(\mathbb{R})
$$

has the following properties:

(i) $\left\|S(t) u_{0}-S(t) v_{0}\right\|_{L^{1}(\mathbb{R})} \leq\left\|u_{0}-v_{0}\right\|_{L^{1}(\mathbb{R})}$ for all $u_{0}, v_{0} \in L^{1}(\mathbb{R})$,

(ii) $S(t)$ maps $L^{1}(\mathbb{R}) \cap B V(\mathbb{R})$ into itself, and $T V\left(S(t) u_{0}\right) \leq T V\left(u_{0}\right)$ for all $u_{0} \in$ $L^{1}(\mathbb{R}) \cap B V(\mathbb{R})$.

And finally, we have:

Theorem 6.2. Assume $u_{0} \in L^{\infty}(\mathbb{R}), a \in W^{1, \infty}\left(\mathbb{R} \times \mathbb{R}_{+}\right)$and $f$ is a $\mathcal{C}^{2}$-function. Then problem (1) has a unique entropy solution $u \in L^{\infty}(\mathbb{R} \times(0,+\infty))$ and

$$
\|u(\cdot, t)\|_{L^{\infty}(\mathbb{R})} \leq\left\|u_{0}\right\|_{L^{\infty}(\mathbb{R})}
$$

for almost all $t \geq 0$. Moreover, if $u_{0} \in L^{\infty}(\mathbb{R}) \cap B V(\mathbb{R})$, then $u(\cdot, t) \in B V(\mathbb{R})$ and $T V(u(\cdot, t)) \leq T V\left(u_{0}\right)$. $5]$.

The proofs of all these results are exactly the same to those of [4: Chapter II, Section

Remark 6.1. For sake of simplicity this paper has been written in the case of one space dimension but the previous results can be extended to the case of any space dimension with no more difficulties. 


\section{References}

[1] Bernard, S.: Lois de Conservation Scalaires à Coefficients Discontinus. Thesis. Guadeloupe: Univ. des Antilles et de la Guyane 1999.

[2] Brezis, H.: Analyse fonctionnelle: théorie et applications. Paris: Masson 1986.

[3] Bouchut, F. and F. James: One-dimensional transport equations with discontinuous coefficients. Nonlin. Anal. TMA 32 (1998), 891 - 933.

[4] Godlewski, E. and P. A. Raviart: Hyperbolic Systems of Conservation Laws (Math. \& Appl.). Paris: Ellipses 1991.

[5] Godlewski, E. and P. A. Raviart: Numerical Approximation of Hyperbolic Systems of Conservation Laws (Appl. Math. Sci.: Vol. 118). New York: Springer 1996.

[6] Hörmander, L.: Lectures on Nonlinear Hyperbolic Differential Equations (Math. and Appl.: Vol. 26). Heidelberg - New York: Springer-Verlag 1997.

[7] Kruzkov, S. N.: First-order quasilinear equations in several independent variables (in Russian). Mat. Sb. 123 (1970), 228 - 255; English transl. in: Math. USSR Sb. 10 (1970), $217-243$.

[8] Lions, J. L. and E. Magenes: Problèmes aux Limites Non Homogènes et Applications (Travaux et Rech. Math.). Paris: Dunod 1968.

[9] Serre, D.: Systèmes de Lois de Conservation: Hyperbolicité, Entropies, Ondes de choc. Paris - New York - Amsterdam: Fondations, Diderot Editeur, Arts et Sciences 1996.

[10] Smoller, J.: Shock Waves and Reaction-Diffusion Equations. New York: Springer-Verlag 1983.

Received 07.12.1999; in revised form 03.04.2001 